See discussions, stats, and author profiles for this publication at: https://www.researchgate.net/publication/353608868

\title{
Design and Re-engineering of an Automated Solar Lawn Mower
}

Conference Paper · July 2021

DOI: 10.1109/ACMI53878.2021.9528175

\section{CITATIONS}

0

7 authors, including:

Joydev Karmakar Rahul

Rajshahi University of Engineering \& Technology

1 PUBLICATION 0 CITATIONS

SEE PROFILE

Nabila Khayer

Rajshahi University of Engineering \& Technology

2 PUBLICATIONS 0 CITATIONS

SEE PROFILE
READS

59

2. Souvik Chakraborty

Rajshahi University of Engineering \& Technology

2 PUBLICATIONS OCITATIONS

\section{SEE PROFILE}

Ar-Rafi Hriday

Rajshahi University of Engineering \& Technology 1 PUBLICATION 0 CITATIONS

SEE PROFILE

Some of the authors of this publication are also working on these related projects:

Service Rate at Customer Care Centre View project 


\section{Design and Re-engineering of an Automated Solar Lawn Mower}

\author{
Joydev Karmakar Rahul \\ Department of Industrial \& Production \\ Engineering \\ Rajshahi University of Engineering \& \\ Technology \\ Kazla, Rajshahi-6204, Bangladesh \\ joyd3v@gmail.com \\ Ar-Rafi Hriday \\ Department of Industrial \& Production \\ Engineering \\ Rajshahi University of Engineering \& \\ Technology \\ Kazla, Rajshahi-6204, Bangladesh \\ arrafihriday@gmail.com
}

\author{
Souvik Chakraborty \\ Department of Industrial \& Production \\ Engineering \\ Rajshahi University of Engineering \& \\ Technology \\ Kazla, Rajshahi-6204, Bangladesh \\ 1605001@student.ruet.ac.bd \\ Md. Limon Mia \\ Department of Industrial \& Production \\ Engineering \\ Rajshahi University of Engineering \& \\ Technology \\ Kazla, Rajshahi-6204, Bangladesh \\ limon.ruet.ipe@gmail.com \\ Toukir Ahmed \\ Lecturer, Department of Industrial \& \\ Production Engineering \\ Rajshahi University of Engineering \& \\ Technology \\ Kazla, Rajshahi-6204, Bangladesh \\ toukir.ahmed@ipe.ruet.ac.bd
}

\author{
Nabila Khayer \\ Department of Industrial \& Production \\ Engineering \\ Rajshahi University of Engineering \& \\ Technology \\ Kazla, Rajshahi-6204, Bangladesh \\ khayer1706@gmail.com \\ Md. Zahidul Islam \\ Assistant Professor, Department of \\ Industrial \& Production Engineering \\ Rajshahi University of Engineering \& \\ Technology \\ Kazla, Rajshahi-6204, Bangladesh \\ zahid@ipe.ruet.ac.bd
}

\begin{abstract}
The study focuses on introducing the design of an efficient automated solar lawn mower. This autonomous vehicle is equipped with sensory unit to avoid obstacles, houses a powerful solar panel along with solar tracking system and carries an electromagnetic trimmer which cuts grass. The model of the mower passed through several inspection simulations to refine and perfect its design. The concern of the end users were taken into consideration and several technical analysis including Kano model assessment, Quality Function Deployment, Function Structure, Bill of Materials, Material Selection and Manufacturing Process Selection were used as the baseline for making any decision regarding the lawn mower. The product features were selected based on the Kano Model of customer need assessment. The Quality Function Deployment converted the customer needs into technical requirements which helped in developing the function structure. Later the function structure aided in designing the product and accelerated the selection of materials and manufacturing processes. The purpose of this project is to assemble the necessary building blocks to develop a less costly, durable, portable, driverless, safer and reliable lawn mowing vehicle.
\end{abstract}

Keywords—lawn mower, trimming mechanism, solar tracker, digital logic method, stress analysis.

\section{INTRODUCTION}

Lawn mowing is an important task to keep the environment aesthetic and decorated. To keep our habitat neat and clean it is essential to curtail the growth of grass after a certain interval of time. Mowing a land is a very hard and time consuming task by the traditional methods. Using traditional methods for lawn mowing for an increased time period can lead to serious Musculoskeletal Disorders (MSD) in the long run. Besides, in today's world where time is absolutely a precious thing, spending time in lawn mowing is not a wise alternative at all. An automated lawn mower can solve this problem. But most of the available models are highly expensive for general people. Also, they are not energy efficient and safe enough. Our study targets to overcome these limitations.

In the old days, sickle and scythe were used to cut grass. Then mechanical cutters were introduced which were driven by human labour. Soon these mechanical cutters were replaced by ride able gasoline lawn mowers. Further enhancements came in the form of electrical lawn mower which also required human assistance. Finally, the concept of robotic lawn mowers was introduced in this era.

This paper describes a portable, safer, more efficient and autonomous lawn mower which can solve the problems of increased hard work and time consumption. The device designed looks mostly like a mini four wheeler with a cutting mechanism using electromagnetic trimmer, a bend solar panel mounted on the top for charging batteries and a proximity sensor on board for detecting any obstacles. The device will run through the lawn and cut grass along the way and at the same time look for solid obstacles. If the sensors detect any blockings in the path, the device will stop immediately and take a 180 degree turn to the next line and continue cutting throughout the field.

\section{LITERATURE REVIEW}

A recent model of an automatic lawn mower imitated human feet in mowing and used crawler tracker. It has been 
made to function by phone [1]. Another design approach [2] utilizes the push force of the human operator while walking and redirects it to the blades through the gear system. Cutting height could be adjusted easily and consisted of wheel, spiral cutter, bed knife, handle. Bevel gear had been used to increase cutting speed. Some designs of manually operated lawn mower included dc electric motor with speed multiplication pulleys and a lift mechanism to cut the grass at an adjustable height, collapsible blades for reducing wear and collection bag to collect grass [3]. Some researchers reviewed the processes for making a more efficient and cost effective lawn mower technology. They compared between internal combustion engine, solar energy and dc or ac source for power generation [4]. Some autonomous mowers use image sensors to detect grass and activate the blades to mow the lawn without any human assistance [5]. A prototype of a robotic lawn mower was made which could navigate on a grass area without having to mount a binding cable at the corners. It used a combination of camera, GPS and ultrasound sensors [6]. Sometimes designers have to tradeoff among several features to fulfill a singular objective. For example the design of a hand held grass cutter traded durability and strength for increasing battery life [7]. The automatic lawn mowers are the possible replacements for the old generation gasoline lawn mowers. The uses of these lawn mowers have the potential to uphold the environment at a decreased operating cost [8]. The advancement of hybrid power source ensures endurance and maximizes operation time while reducing the necessity of direct electric charging. Bladeless design of the cutting head has lowered the chances of severe injuries [9]. But the risk factor is still there as the cutting head uses rotating plastic/metal stripes that are mounted at the front. This is due to the lack of obstacle sensing units.

A quick comparison on various models of modern lawn mower reveals a handful of opportunities to improve over previous designs. The papers that we came across mostly promoted a single feature as their methodologies were structured to do so. They never showed any commercial interest for manufacturing. So the suggested designs never went through critical stages like- the market and industrial analysis. This study demonstrates all the steps of improving a lawn mower starting from a customer survey to selecting the most cost efficient means of mass production considering the requirements of safety and overall increase in product efficiency. Besides, this study focused on improving the design aspects and suggests an efficient lawn mower model that uses renewable energy, automatic in nature, equipped with cutting-edge trimming mechanism with a less costly chassis.

\section{Methodology}

The methodology part is divided into ten sections. They are described below:

Firstly, a sample of 200 individuals was randomly chosen to mitigate the chances of bias during the survey and to get a general idea on customer needs regarding the market or product. Then these needs were converted using Kano model and QFD respectively. The Kano Model categorized these needs into several attributes and isolated attractive features to pay extra attention on them. And the QFD worked as a translation tool which translated the customer requirements into technical ones. The insight obtained from previous sections helped to establish the function structure which in turn developed the component relation diagram to show the interactions between several components. Then a $3 \mathrm{D}$ model of the product was created to define the initial design. The 3D model demonstrated the aesthetic and design aspects of the product and helped to quantify the number of assemblies and sub-assemblies leading to the genesis of BOM. After that the materials were chosen using the Digital Logic Method based on availability, usability and functions to save the cost of expensive prototyping. The previously made 3D model was tested under various loading conditions using computer simulation and minor modifications were made accordingly. Finally the best suited process for manufacturing the parts in bulk quantity was chosen. The factors considered for the manufacturing process selection were to utilize resources and optimize operations.

\section{A. Survey}

A survey was performed on 200 people comprising gardeners and farmers (39\%), shopkeepers and businessmen $(16 \%)$, engineers $(10 \%)$, students $(8 \%)$, teachers $(7 \%)$ and others $(20 \%)$ to get an idea of the features and specifications that customer want in a lawn mower. A general overview and advantages of an automated lawn mower were described in the survey questionnaire. The respondents of the survey were mainly those who are need of a lawn mower to keep their lawn clean. The survey comprised of 17 closed end questions and 1 open end question. The findings of the survey along with the relative importance of the customer requirements are listed in TABLE I.

TABLE I RELATIVE IMPORTANCE

\begin{tabular}{|c|c|}
\hline Customer Requirements & Relative Importance \\
\hline Portable & 7 \\
\hline High speed & 5 \\
\hline Hybrid Power (AC+Solar) & 30 \\
\hline Grass Collector & 7 \\
\hline Attractive & 9 \\
\hline Adjustable Height & 10 \\
\hline Splash Proof & 6 \\
\hline Less Weight & 8 \\
\hline Cheap & 9 \\
\hline Easy to Operate & 10 \\
\hline Safety & 9 \\
\hline Long Service life &
\end{tabular}

Hybrid Power Source, Splash proof and Safety of Operation were the requirements with the highest relative importance whereas grass collector had the lowest importance.

\section{B. Kano Model}

The Kano model is a five-category classification system for consumer expectations in product creation and customer satisfaction. They are: Must-be, One dimensional, Attractive, Indifferent and Reverse. If the absence of a consumer need in terms of a product attribute results in complete disappointment and the presence of the need does not improve satisfaction, then the need is called Must-be. If 
the fulfillment of an attribute increases happiness and vice versa then it is called one dimensional. An attribute is said to be attractive if it contributes to increased satisfaction, although it is not supposed to be in the product. These functions will make this product more desirable and different than existing ones. When the presence or absence of a function has no effect on satisfaction, it is called indifferent. Lastly when the presence of an attribute induces disappointment, it is called reverse attribute [10]. All the attributes of the product are listed in TABLE II.

TABLE II KANO MODEL OF ATTRIBUTES

\begin{tabular}{|c|c|c|}
\hline Serial no. & Category & Attributes \\
\hline 1 & Must be & $\begin{array}{c}\text { Splash proof, Hybrid Power Source, } \\
\text { Eco Friendly, Long Working Hour }\end{array}$ \\
\hline 2 & One-dimensional & $\begin{array}{c}\text { Easy to Operate, Durable, Portable, } \\
\text { Safe }\end{array}$ \\
\hline 3 & Attractive & $\begin{array}{c}\text { Solar Tracker, Trimmer, Shock } \\
\text { Absorber }\end{array}$ \\
\hline 4 & Indifferent & Chip-set, Sensor Type, Programs \\
\hline 5 & Reverse & Noise, Expense, Size \\
\hline
\end{tabular}

The attractive features of our product are mentioned in below:

\section{Solar tracker:}

The solar panel is mounted at the top of the lawn mower with the tracking unit. The solar tracker tracks the movement of the sun and redirects the solar tray towards the sun accordingly.

\section{Trimmer:}

The product comes with a trimming mechanism rather than conventional rotary blades. This feature ensures safety and increases the aesthetics.

\section{Shock absorber:}

There was no physical shock absorber included in the lawn mower. The solar tray is allowed to have a limited motion around the pivot axis. This constant movement absorbs the shock generated by the rough surface.

These three (3) features are unique to our model and separates existing similar products from our proposed lawn mower design.

\section{Quality Function Deployment (QFD)}

QFD is a method for evaluating a producer's ideas to best meet a consumer need. So, it's the 'voice of the consumer,' often referred to as the 'House of Quality.' It listens to the customer's needs and prioritizes them in order to identify the customer's appropriate area of interest in the eyes of a technical team. In a generic diagram of QFD there are six parts and they are Customer requirements, Technical requirements, Co-relation matrix, Relation matrix, Benchmarking and Engineering measures [11].

The features that a consumer really demands from a product are known as customer requirements. These desires are the customer's actual expression. Technical specifications, also known as the "voice of the manufacturer," are the response to a customer's request. There are technical standards that the technical team has developed in order to satisfy the needs of customers. Corelation matrix is a roof shape triangular box at the top of the house of quality containing a matrix. Each matrix represents the co-relation between two technical requirements. These relationships are denoted by the following symbols: Strongly positive $(++)$, Positive $(+)$, Neutral( . ), Negative(-), Strongly Negative(--). The relation matrix showcases the relation between customer and technical requirements. The level of impact is symbolized by: Strongly related (9), Moderately related (3), Weakly related (1), None (0). Fig. 1 shows the QFD of the product.

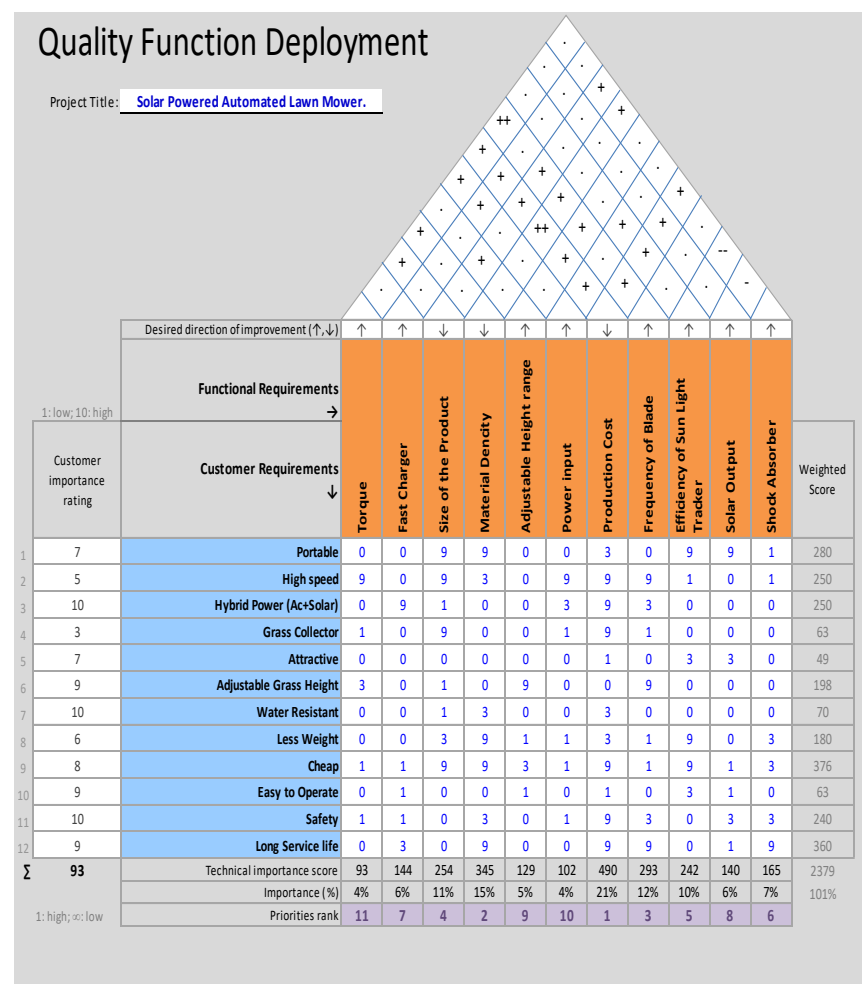

Fig. 1. Quality Function Deployment

The calculations of the QFD data yields the priorities order of the technical requirements to meet the customer requirements. The order is given as: 1. Product cost, 2. Material density, 3. Frequency of the blade, 4. Size of the product, 5. Efficient sun light tracker, 6. Shock Absorber, 7. Fast charger, 8. Solar output, 9. Adjustable height, 10. Power input capacity, 11. Torque.

\section{Function Structure}

The automated solar lawn mower has a main function which is cutting grasses. It uses information power and force to perform this function. For better understanding the main function was divided into four sub functions (Fig. 2). The first sub function uses power and information to guide the tires of the lawn mower. The second function senses obstacles and creates a feedback loop of information which is used as an input for the 1 st and 2 nd sub functions. The purpose of these sub function is to control the electromagnetic mechanism of the trimmer blade to cut the grass at a prefixed size and uses power and information in the 
process. And the fourth and final sub function is the uniaxial solar tracker which positions the solar panel mounted above the lawn mower toward the sun to maximize the power generation.

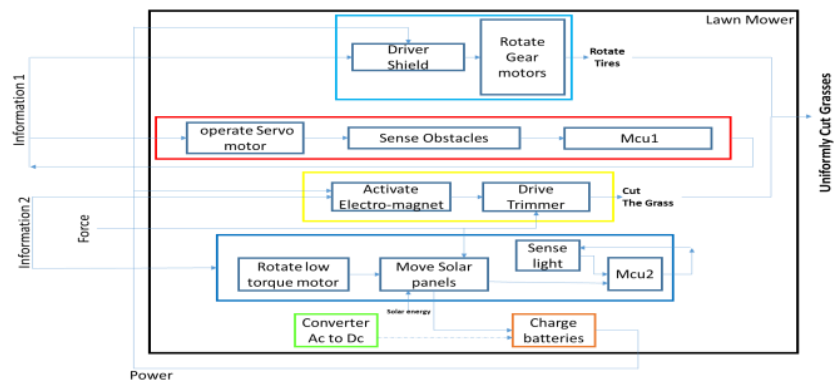

Fig. 2. Function Structure of Lawn Mower

\section{E. Component Relation Diagram}

The component relation diagram view of the lawn mower (Fig. 3) has shown two micro controlling units namely microcontroller-1 and microcontroller-2. The unit-1 is connected to servo motor, proximity sensor, electromagnetic trimmer, motor driver shield and battery packs. The main purpose of unit-1 is to control the driving mechanism in accordance of the proximity sensors and power the electromagnet.

Unit-2 is dedicated to solar tracking. It houses a low torque motor light intensity sensors and solar cells. The battery gets power from solar and as well as from the charge through voltage regulation unit.

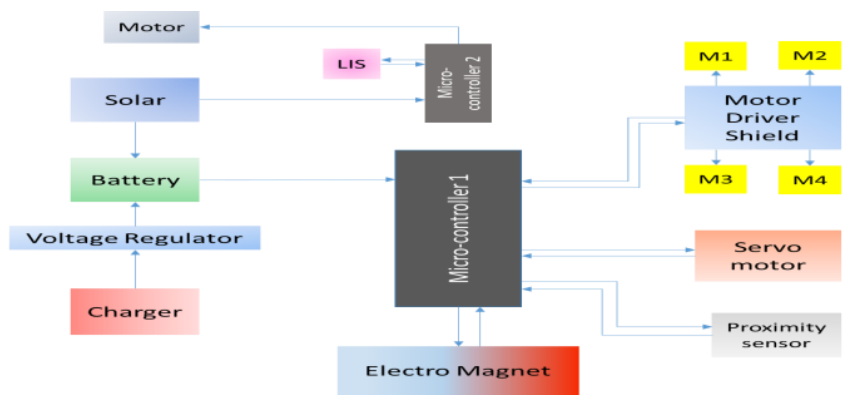

Fig. 3. Component Relation Diagram

\section{F. 3D Modelling}

Fig. 4 shows the exploded view of the lawn mower. Here the parts are disassembled to make more sense and better visualize the design components.

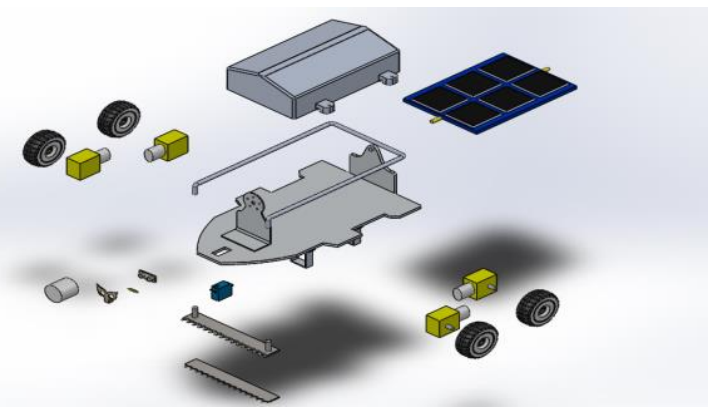

Fig. 4. Exploded view

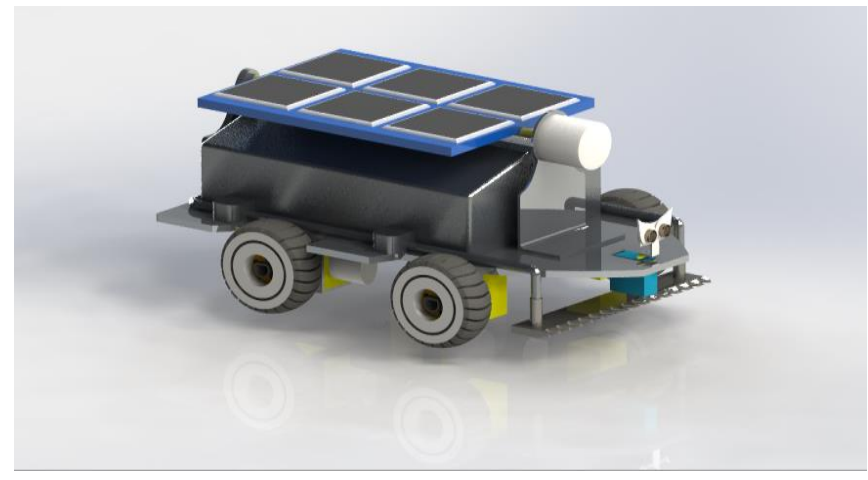

Fig. 5. 3D Model of Lawn Mower

In the simulation environment we have added materials to the respective parts and rendered the assembly model which generated the 3D view in the Fig. 5.

\section{G. Bill of Materials(BOM)}

A bill of material is a source of information of the raw material, sub-assemblies, intermediate assemblies, subcomponents and quantities of each required to manufacture an end product. In this context, there are five main parts of the assembly namely- 1) Body, 2) Solar System, 3) Drive System, 4) Power System and 5) Trimmer. TABLE V shows the components of each part of the assembly along with their quantities required.

TABLE III BILL OF MATERIALS (BOM)

\begin{tabular}{|c|c|c|}
\hline \multicolumn{2}{|c|}{ Sub-assembly name } & Quantity \\
\hline \multirow{6}{*}{ Body } & Base & 1 \\
\hline & Vertical Holder 1 & 1 \\
\hline & Vertical Holder 2 & 1 \\
\hline & Trimmer Holder & 1 \\
\hline & Enclosure & 1 \\
\hline & Clamp & 4 \\
\hline \multirow{6}{*}{ Solar System } & Solar Panel & 3 \\
\hline & Motor & 1 \\
\hline & Printed Circuit Board & 1 \\
\hline & LDR & 2 \\
\hline & Solar Holder & 1 \\
\hline & Solar Mounter & 1 \\
\hline \multirow{5}{*}{ Drive System } & Servo Motor & 1 \\
\hline & Arduino UNO & 1 \\
\hline & Proximity Sensor & 1 \\
\hline & Gear Motor & 4 \\
\hline & Wheel & 4 \\
\hline \multirow{5}{*}{ Power System } & Charger & 1 \\
\hline & Battery & 4 \\
\hline & Charging Circuit & 1 \\
\hline & Indicator LED & 3 \\
\hline & On/Off Switch & 3 \\
\hline \multirow{4}{*}{ Trimmer } & Blade & 1 \\
\hline & Holder Support & 1 \\
\hline & Electro Magnet & 1 \\
\hline & Pulse Circuit & 1 \\
\hline \multirow{2}{*}{ Others } & Screw & 24 \\
\hline & Wires & - \\
\hline
\end{tabular}




\section{H. Material Selection}

The step in a design process in which materials of different parts of a product are selected considering the work environment implications is material selection. The material has to be selected in such a way that the overall cost of the product is minimized and the performance goals are met. Material selection is done by comparing the cost and properties of the candidate materials. Digital Logic Method was used to select the material of this product. Different candidates were chosen at the start of the selection process of which the selected ones by Digital Logic Method are listed in TABLE III based on the highest value of performance index. Factors considered for the selection process were- 1) Hardness, 2) Density, 3) Specific Heat, 4) Yield Strength, 5) Expansion, 6)Young's Modulus.

TABLE IV LIST OF MATERIALS

\begin{tabular}{|c|c|c|}
\hline Part & Sub-assembly & Material \\
\hline \multirow{2}{*}{ Body } & Chassis & \multirow{2}{*}{ Al alloy } \\
\cline { 2 - 2 } & Enclosure & \multirow{2}{*}{ Stainless Steel } \\
\cline { 2 - 2 } Trimmer & Upper & \\
\cline { 2 - 2 } & Lower & \\
\hline
\end{tabular}

\section{Stress Analysis}

Stress analysis is a method to determine the stresses and strains in materials and structures subjected to forces [12]. The starting point for stress analysis are- geometrical description of the structure, the properties of the materials used for its parts, how the parts are joined and the maximum or typical forces that are expected to be applied to the structure. The output data is typically a quantitative description of how the applied forces spread throughout the structure, resulting in stresses, strains and the deflections of the entire structure.

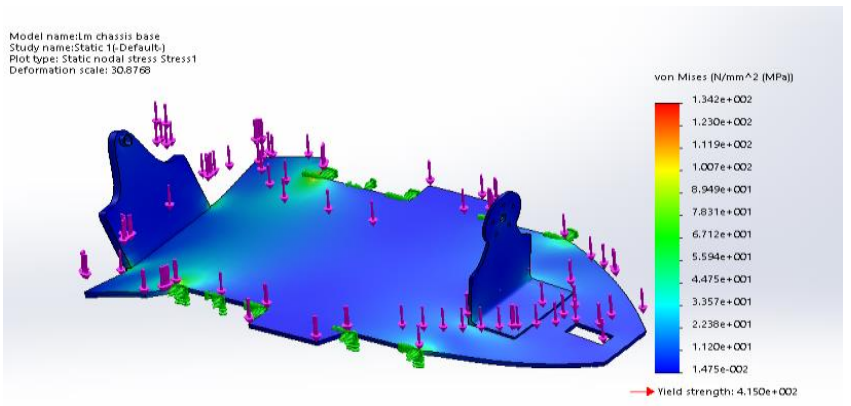

Fig. 6. Stress Analysis for Chassis

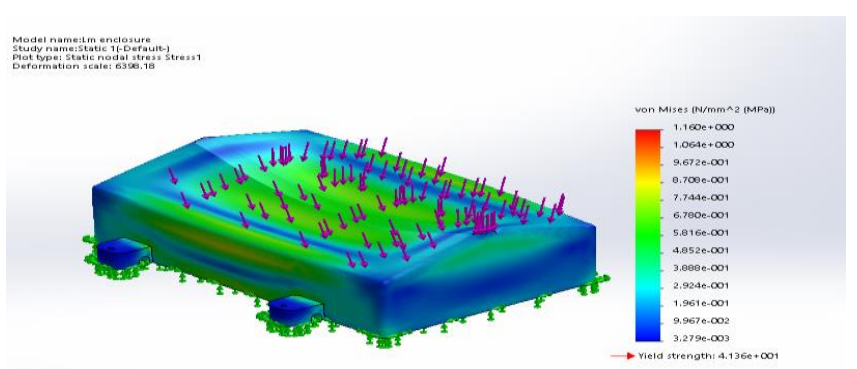

Fig. 7. Stress Analysis for Enclosure

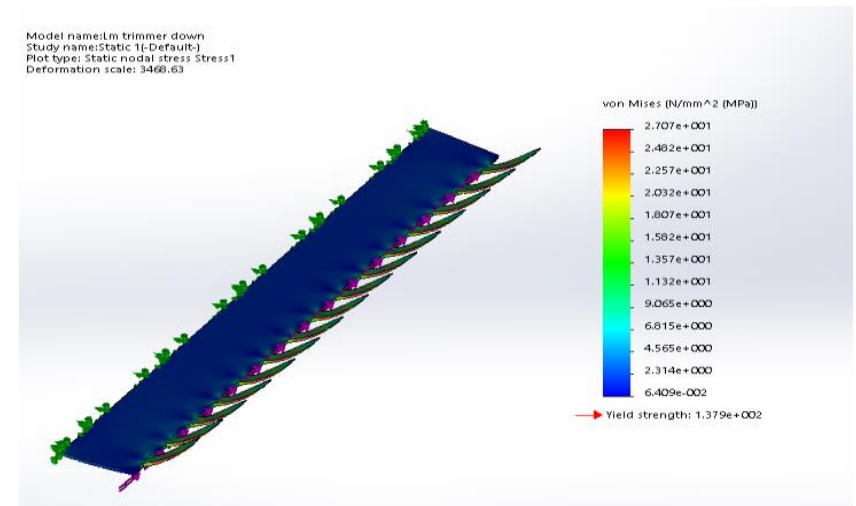

Fig. 8. Stress Analysis for Trimmer

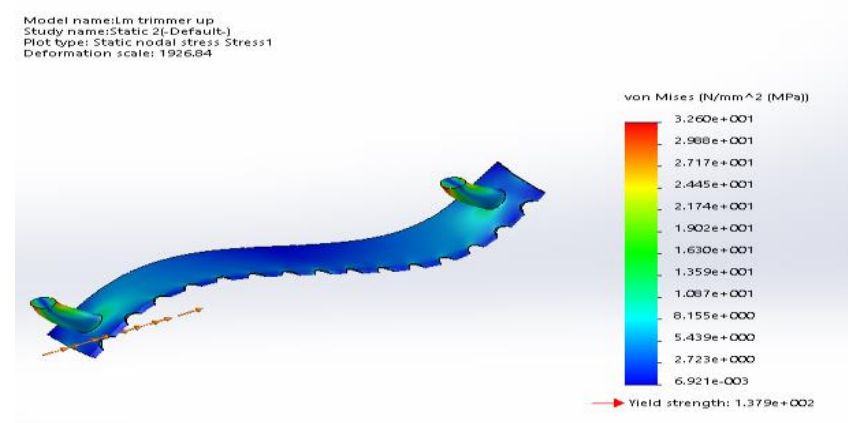

Fig. 9. Stress Analysis for Trimmer Holder

Stress analysis was performed for our product 'Automated Solar Lawn Mower' in Solidworks Static Simulation Environment. The stress properties of three parts- the chassis, enclosure and trimmer were simulated using Von Mises method. The green arrows indicate the points of fixtures while the purple arrows represent the points and direction of applied forces. Here, in all three cases the maximum induced stress hardly passed the green section of the spectrum which indicates the design of the sub-assemblies passed the inspection. Fig. 6 to Fig. 9 shows the stress analyses of different parts.

\section{J. Manufacturing Process Selection}

The process of selecting the most efficient and cost effective process for mass production is known as manufacturing process selection. The manufacturing process of the product was also selected by Digital Logic Method. TABLE IV lists the processes for cutting and joining of different parts of the product. Factors considered for the selection process were- 1) Cost, 2) Power Requirement, 3) Dimensional Accuracy, 4) Strength, 5) Surface Finish, 6) Processing Time

\section{TABLE V LIST OF MANUFACTURING PROCESSES}

\begin{tabular}{|c|c|c|}
\hline \multirow{2}{*}{ Sub-assembly } & \multicolumn{2}{|c|}{ Process } \\
\cline { 2 - 3 } & Cutting & Joining \\
\hline Chassis & Blanking & TIG \\
\hline Enclosure & Blanking & TIG \\
\hline Upper & Blanking & Fastening \\
\hline Lower & Blanking & Fastening \\
\hline
\end{tabular}




\section{DISCUSSION}

The main function of the product is grass cutting in gardens, lawns or yards. By analyzing the survey, rank of customer requirements were found. These were safety, splash proof, hybrid power (Ac \& Solar). Knowing these requirements was really beneficial in the earlier stages of product development. The attractive features from the Kano Model were mainly kept in focus in this study. Solar tracker, shock absorber, trimmer has added a new value to the automated lawn mower. QFD helped finding the order priority of the technical requirements to meet the customer requirements. From Bill of Material (BOM), the quantities of each component required were known. Digital logic method was used to select Al alloy and Stainless Steel for body and trimmer part according to the highest performance index. In manufacturing process selection, blanking process was selected for aluminum alloy. The use of this product speeds up the method of grass cutting by reduction of time to cut. In addition, it is less expensive, light weight, environmental friendly and is helpful for non-commercial use (home users) in parks, houses or yards to cut grass.

\section{CONCLUSION}

The most important technical requirements from the QFD are price and weight of the product. The unit cost of the product is far lesser than the current market price. To make the object lighter Aluminum Alloy was used in most parts of the product. The safety of the lawn mower was a major cause of concern particularly for the children. Children were injured in many cases due to the rotating blade of the trimmer. This problem has been solved in the study by the inclusion of electromagnetic trimming mechanism which led to an increased safety. Most of the models available in the market do not have a hybrid source for power generation let alone a solar tracker. To maximize the power generation and to improve overall efficiency, capacity solar tracker was introduced to the system. The solar panel charged the batteries of the mower without having to manually charge them using an AC power source. So this design eliminates the most of the shortcomings of the previous designs. However, the product has some drawbacks. Grass collection box was excluded from the design to make it more portable. The GPS was not installed. In this model, it was not possible to incorporate the optical sensor for grass recognition system as it requires extensive research which was beyond the scope of this paper. The prototype lacks the homing device which may lead to the off-coursing of the lawn mower.

\section{REFERENCES}

[1] J.-C. Liao, S.-H. Chen, Z.-Y. Zhuang, B.-W. Wu and Y.-J. Chen, "Designing and Manufacturing of Automatic Robotic Lawn Mower," Processes, vol. 9, p. 358, 2021.

[2] N. Nagarajan, N. S. Sivakumar and R. Saravanan, "Design and fabrication of lawn mower," Asian Journal of Applied Science and Technology (AJAST), vol. 1, p. 50-4, 2017.

[3] B. E. Okafor, "Simple design of self-powered lawn mower," 2013.

[4] P. P. Dutta, A. Baruah, A. Konwar and others, "A technical review of lawn mower technology," ADBU Journal of Engineering Technology, vol. 4, 2016.

[5] A. R. Reddy, N. V. Chaitanya, P. Abhishek, A. Suvarnamma and K. SRMIST, "Autonomous Solar Based Lawn Mower," International Journal of Pure and Applied Mathematics, vol. 119, p. 1312913134, 2018.

[6] J. M. Derander, P. Andersson, E. Wennerberg, A. Nitsche, E. Moen and F. Labe, "Smart robot lawn mower," Department of Computer Science and Engineering Chalmers University of Technology University of Gothenburg Gothenburg, Sweden, 2018.

[7] R. Sivagurunathan, L. Sivagurunathan and J. C. J. Hao, "Design and fabrication of low cost portable lawn mower," Scholars Journal of Engineering and Technology, vol. 5, p. 584-591, 2017.

[8] S. V. Tanaji, C. S. Chandrakant, P. S. Shashikant, G. O. Raju and G. S. Bhalchandra, "Automated mower robo," Int. Res. J. of Eng. and Technol, vol. 5, 2018.

[9] D. A. Aponte-Roa, X. Collazo, M. Goenaga, A. A. Espinoza and K. Vazquez, "Development and evaluation of a remote controlled electric lawn mower," in 2019 IEEE 9th Annual Computing and Communication Workshop and Conference (CCWC), 2019.

[10] “Quality Function Deployment(QFD)". Accessed on: Jun. 8, 2021. [Online]. Available: https://quality-one.com/qfd/

[11] "Kano Analysis". Accessed on: Jun. 8, 2021. [Online].Available: https://sapioresearch.com/kano-analysis

[12] "Stress analysis". Accessed on: Jun. 13, 2021. [Online].Available: https://simple.m.wikipedia.org/wiki/Stress_analysis 